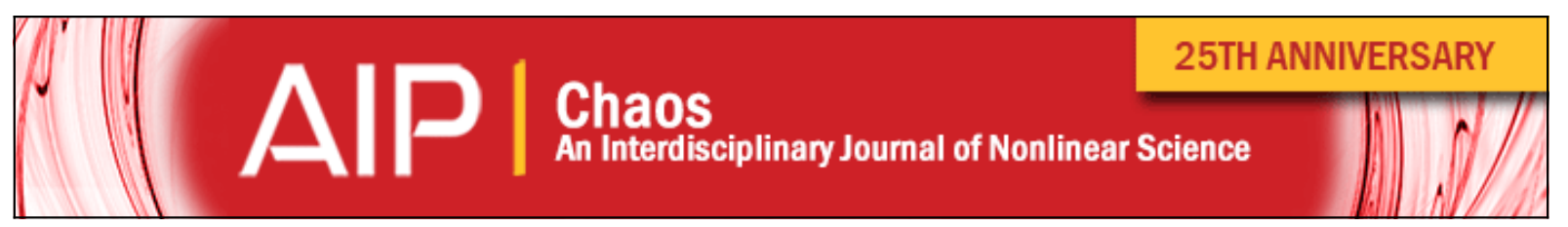

\title{
Analysis of noise-induced transitions from regular to chaotic oscillations in the Chen system
}

Irina Bashkirtseva, Guanrong Chen, and Lev Ryashko

Citation: Chaos: An Interdisciplinary Journal of Nonlinear Science 22, 033104 (2012); doi: 10.1063/1.4732543

View online: http://dx.doi.org/10.1063/1.4732543

View Table of Contents: http://scitation.aip.org/content/aip/journal/chaos/22/3?ver=pdfcov

Published by the AIP Publishing

\section{Articles you may be interested in}

The effect of noise on the complete synchronization of two bidirectionally coupled piecewise linear chaotic systems

Chaos 19, 013131 (2009); 10.1063/1.3080194

Constructive Technique of Noise Sensitivity Analysis for Regular and Chaotic Nonlinear Systems AIP Conf. Proc. 922, 491 (2007); 10.1063/1.2759727

Complete and generalized synchronization in a class of noise perturbed chaotic systems Chaos 17, 023106 (2007); 10.1063/1.2718491

Noise-Induced Transition and Synchronization in Paddlefish Electroreceptors AIP Conf. Proc. 665, 150 (2003); 10.1063/1.1584886

Analytical and numerical studies of noise-induced synchronization of chaotic systems Chaos 11, 665 (2001); 10.1063/1.1386397

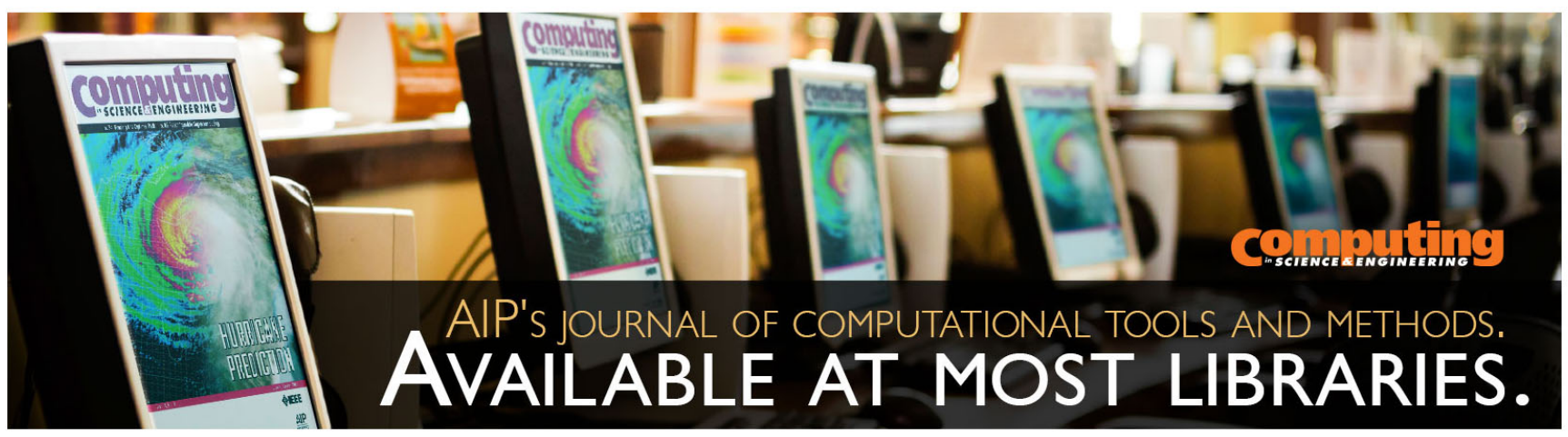




\title{
Analysis of noise-induced transitions from regular to chaotic oscillations in the Chen system
}

\author{
Irina Bashkirtseva, ${ }^{1}$ Guanrong Chen, ${ }^{2}$ and Lev Ryashko ${ }^{1}$ \\ ${ }^{1}$ Department of Mathematics, Ural State University, Lenina, 51, Ekaterinburg, Russia \\ ${ }^{2}$ Department of Electronic Engineering, City University of Hong Kong, Hong Kong, \\ People's Republic of China
}

(Received 23 March 2012; accepted 18 June 2012; published online 5 July 2012)

\begin{abstract}
The stochastically perturbed Chen system is studied within the parameter region which permits both regular and chaotic oscillations. As noise intensity increases and passes some threshold value, noiseinduced hopping between close portions of the stochastic cycle can be observed. Through these transitions, the stochastic cycle is deformed to be a stochastic attractor that looks like chaotic. In this paper for investigation of these transitions, a constructive method based on the stochastic sensitivity function technique with confidence ellipses is suggested and discussed in detail. Analyzing a mutual arrangement of these ellipses, we estimate the threshold noise intensity corresponding to chaotization of the stochastic attractor. Capabilities of this geometric method for detailed analysis of the noiseinduced hopping which generates chaos are demonstrated on the stochastic Chen system. (C) 2012 American Institute of Physics. [http://dx.doi.org/10.1063/1.4732543]
\end{abstract}

Noise-induced chaos in stochastically perturbed nonlinear deterministic systems with regular attractors has been studied extensively. This phenomenon has received a great deal of interest among researchers from various fields of physics, life sciences, and engineering. Nonlinearity, even in a non-chaotic regime, can imply a non-uniformity of phase portraits, diverse forms of coexicting regular attractors with a complicated geometry of basins of attraction. Under random disturbances, a phase trajectory can cross separatices and induce stochastic hopping between both coexisting regular attractors and their different but close portions. As a consequence of this hopping, the random trajectories with a high probability fall within zones of divergency (local instability), and the dynamics of the perturbed system as a whole becomes chaotic. The positivity of the largest Lyapunov exponent can be used as a measure that this new noise-induced regime is chaotic.

In this paper, for the constructive prediction of the noise-induced transitions from regular to chaotic oscillations in a dynamical system, a new stochastic sensitivity function technique is developed and applied. This technique enables to find dispersion ellipses of the random states for the stochastically perturbed dynamical system around deterministic attractors. The sizes of these ellipses are enlarged as noise intensity increases. The constructive method of the dispersion ellipses allows to estimate the threshold of the noise intensity corresponding to the deformation of a regular stochastic attractor to a chaotic one. The effectiveness of this approach is demonstrated by the stochastically perturbed Chen system.

\section{INTRODUCTION}

Analysis of the interplay between stochasticity and nonlinearity in dynamical systems is a fundamental problem in both theoretical statistical physics and modern engineering applications.
In nonlinear systems, noise can induce various phenomena which have no analogies in the deterministic setting. ${ }^{1,2}$ New stochastic regimes such as noise-induced transitions, ${ }^{3,4}$ stochastic bifurcations, ${ }^{5-7}$ stochastic resonance, ${ }^{8,9}$ noiseinduced order, ${ }^{10,11}$ noise-induced chaos, ${ }^{12-14}$ noise-induced excitability ${ }^{15,16}$ and intermittency, ${ }^{17-19}$ noise-induced crises, ${ }^{20,21}$ noise-induced oscillations (coherent resonance), ${ }^{22-24}$ and noise-induced unstable dimension variability ${ }^{25}$ are currently the objects of considerable research.

Due to nonlinearity, even in a non-chaotic regime, the dynamical system can exhibit multiple coexisting regular attractors. These deterministic attractors can be either simple (equilibria) or complicated (limit cycles in period-doubling zone, tori). For small noise, random trajectories are attracted to deterministic attractors, which are localized not far from the basin of the attraction. As the noise intensity increases, random hopping between coexisting attractors and their basins of attraction is observed with a high probability. ${ }^{26-28}$ This hopping can generate noise-induced chaos.

But noise-induced chaotization can be observed in the monostable systems also. Indeed, for nonlinear system with single attractor in a sufficiently complicated spatial form, the stochastic hopping between its different but close portions can be induced. ${ }^{29-31}$ As a consequence of this hopping, the dynamics of the perturbed system geometrically become chaos-like. The change of the phase space arrangement of the correspondent stochastic attractor is associated with the change of its dynamical properties. As the noise intensity is increased from zero and passes through some threshold value, the largest Lyapunov exponent becomes positive. The positivity of the Lyapunov exponent justifies that this new noise-induced regime is chaotic dynamically. A scaling analysis of the Lyapunov exponents for various dynamical systems with noise-induced chaotic dynamics is a subject of extensive analysis recently. ${ }^{19,25,30}$

Note that the identification of chaos by analyzing Lyapunov exponents is very costly for long-time direct numerical 
simulations of random trajectories. For the prediction of noise-induced chaotization, moreover, it is desirable to develop an analytical method taking into account the interconnection between the geometrical features of an unperturbed deterministic attractor and its stochastic sensitivity. An approach using quasipotential was proposed in Ref. 32 for scaling analysis of noise-induced chaos. In a recent work, ${ }^{33}$ a new constructive method for the analysis of noise-induced transitions via stochastic sensitivity functions technique was suggested. The effectiveness of this method was illustrated in the study of the transitions between two coexisting limit cycles of the Lorenz system.

In this paper, we extend the stochastic sensitivity functions technique to analyze the probabilistic mechanism of noise-induced chaotization generated by random hopping between different but close portions of a single regular attractor. Specifically, we focus on the case where this attractor is a stable limit cycle. The cycle with arranged nearby parts of its trajectory (e.g., multiple cycle) is an appropriate model for studying the noise-induced hopping mechanism within the single attractor. The familiar deterministic Chen system exhibits a region of period-doubling bifurcations with cycles. ${ }^{34}$ In the present paper, we apply the stochastic sensitivity functions technique and a method of dispersion ellipses to carry out detailed analysis of noise-induced chaotization within that region of the Chen system.

In Sec. II, we present briefly a mathematical background of the technique used for the approximation of the probabilistic distribution and a description of the geometrical features of the stochastically perturbed limit cycles.

In Sec. III, we consider the stochastically perturbed Chen system in the parameter region that admits both regular and chaotic oscillations. We furthermore study the phenomenon of random transitions between close portions of the stochastic cycles. Our constructive method for analyzing these transitions allows to estimate the threshold of noise intensity corresponding to the deformation of a regular stochastic attractor to a chaotic one. We show how this threshold value depends on the multiplicity of stochastically forced cycles.

\section{STOCHASTIC SENSITIVITY FUNCTION FOR 3D CYCLES}

Consider a general deterministic system of nonlinear differential equations,

$$
\dot{x}=f(x) .
$$

Suppose that system (1) has a $T$-periodic solution $\xi(t)$ with an exponentially stable phase curve $\gamma$ (limit cycle). It means that for a small neighborhood $\Gamma$ of the cycle $\gamma$ there exist constants $K>0, l>0$ such that for any solution $x(t)$ of the system (1) with $x(0)=x_{0} \in \Gamma$ the following inequality holds

$$
\|\Delta(x(t))\| \leq K e^{-l t}\left\|\Delta\left(x_{0}\right)\right\| .
$$

Here, $\Delta(x)=x-\gamma(x)$ is a deviation of a point $x$ from a cycle $\gamma, \gamma(x)$ is a point on cycle $\gamma$ that is nearest to $x$.

Along with the unperturbed deterministic system (1), consider an Ito stochastic system

$$
\dot{x}=f(x)+\varepsilon \sigma(x) \dot{w} .
$$

Here, $w(t)$ is an $n$-dimensional Wiener process and $\sigma(x)$ is an $n \times n$ matrix-valued function of perturbation with intensity $\varepsilon>0$.

The random trajectories of the perturbed system (2) leave the closed curve of the deterministic cycle $\gamma$ and, due to cycle stability, form some kind of bundles (stochastic cycles) around it. The stationary density function $\rho(x, \varepsilon)$ describing the probabilistic distribution of random states in this stochastic cycle is governed by the Kolmogorov-FokkerPlanck (KFP) equation. In this paper, we will use asymptotics $^{35}$ of this stationary density function based on the quasipotential $v(x)=-\lim _{\varepsilon \rightarrow 0} \varepsilon^{2} \log \rho(x, \varepsilon)$.

We use Gaussian approximation for the probability density of the bundles of random trajectories, localized near the cycle $^{36}$

$$
\rho \approx K e^{-\frac{v(x)}{\varepsilon^{2}}} \approx K \exp \left(-\frac{\left(\Delta(x), \Phi^{+}(\gamma(x)) \Delta(x)\right)}{2 \varepsilon^{2}}\right)
$$

with the covariance matrix $\varepsilon^{2} \Phi(\gamma)$. Here, "+" means a pseudoinversion. The covariance matrix characterizes the dispersion of the points of intersection on the random trajectories, with a hyperplane orthogonal to the cycle at the point $\gamma$. The function $\Phi(\gamma)$ is a stochastic sensitivity function (SSF) of the limit cycle. This function allows to describe the most essential geometrical features of the probabilistic distribution of the random trajectories around the deterministic cycle.

In this paper, for the function $\Phi(\gamma)$, we use the following parametrization: $\Phi(\xi(t))=W(t)$, where the solution $\xi(t)$ connects the points of the cycle $\gamma$ with the points of an interval $[0, T)$.

Due to the stability of the limit cycle $\gamma$, the matrix function $W(t)$ is the unique solution of the boundary value problem for the Lyapunov equation

$$
\dot{W}=F(t) W+W F^{\top}(t)+P(t) S(t) P(t),
$$

under conditions

$$
\begin{gathered}
W(t) r(t) \equiv 0, \\
W(0)=W(T) .
\end{gathered}
$$

Here,

$$
\begin{aligned}
& F(t)=\frac{\partial f}{\partial x}(\xi(t)), S(t)=\sigma(\xi(t)) \sigma^{\top}(\xi(t)), \\
& r(t)=f(\xi(t)), P(t)=P_{r(t)}, P_{r}=I-r r^{\top} / r^{\top} r,
\end{aligned}
$$

in which $P_{r}$ is the projection matrix onto the subspace orthogonal to the vector $r \neq 0$. It follows from the condition (4) that the matrix $W(t)$ is singular $(\operatorname{det} W(t) \equiv 0)$.

The covariance matrix $\varepsilon^{2} W(t)$ characterizes the dispersion of the points of intersection of random trajectories with hyperplane $\Pi_{t}$ which is orthogonal to the cycle at the point $\xi(t)$. The stochastic sensitivity matrix $W(t)$ is connected here with the orthogonal plane $\Pi_{t}$. Let a plane $\Pi$ be an arbitrary Poincare section at the same point $\xi(t)$. The stochastic 
sensitivity matrix $W_{\Pi}(t)$ corresponding to this Poincare section $\Pi$ can be found as follows:

$$
W_{\Pi}(t)=B(t) W(t) B^{\top}(t), \quad B(t)=I-\frac{r(t) b^{\top}}{r^{\top}(t) b},
$$

where $b$ is a directing vector that is orthogonal to $\Pi$.

\section{A. Case of 3D cycles}

For 3D-cycles, due to the singularity equation (4), the matrix $W(t)$ has the following decomposition:

$$
W(t)=\lambda_{1}(t) v_{1}(t) v_{1}^{\top}(t)+\lambda_{2}(t) v_{2}(t) v_{2}^{\top}(t) .
$$

Here, $\lambda_{1}(t) \geq \lambda_{2}(t) \geq \lambda_{3}(t) \equiv 0$ are the eigenvalues with the corresponding eigenvectors $v_{1}(t), v_{2}(t), v_{3}(t)$ of the matrix $W(t)$. Note that $v_{1}(t), v_{2}(t)$ can be considered as a basis of the plane $\Pi_{t}$, and a vector $v_{3}(t)$ is orthogonal to $\Pi_{t}$.

Our proposed constructive method for computing the elements $\lambda_{1}, \lambda_{2}, v_{1}, v_{2}$ of the decomposition (6) is as follows.

Denote by $u_{1}(t), u_{2}(t)$ some orthonormal basis of the plane that is orthogonal to the cycle at the point $\xi(t)$. One can easily find this basis if $T$-periodic solution $\xi(t)$ is known (see Appendix). The eigenvectors $v_{1}(t), v_{2}(t)$ can be represented by a rotation of the basis $u_{1}(t), u_{2}(t)$ with some angle $\varphi(t)$,

$$
\begin{aligned}
& v_{1}(t)=u_{1}(t) \cos \varphi(t)+u_{2}(t) \sin \varphi(t), \\
& v_{2}(t)=-u_{1}(t) \sin \varphi(t)+u_{2}(t) \cos \varphi(t) .
\end{aligned}
$$

Thus, the decompositions (6) and (7) allow to express an unknown solution $W(t)$ of the systems (3)-(5) in terms of three scalar functions $\lambda_{1}(t), \lambda_{2}(t), \varphi(t)$. A mathematical basis for the algorithms of the calculation $W(t)$ through $\lambda_{1}(t), \lambda_{2}(t), \varphi(t)$ is given by the following theorems proved in Ref. 36.

Theorem 1. The matrix $V(t)$ is a solution of the systems (3)-(4) if and only if the scalar functions $\lambda_{1}(t), \lambda_{2}(t), \varphi(t)$ of the decompositions (6)-(7) satisfy

$$
\begin{aligned}
\dot{\lambda}_{1} & =\lambda_{1} v_{1}^{\top}\left[F+F^{\top}\right] v_{1}+v_{1}^{\top} S v_{1}, \\
\dot{\lambda}_{2} & =\lambda_{2} v_{2}^{\top}\left[F+F^{\top}\right] v_{2}+v_{2}^{\top} S v_{2}, \\
\left(\lambda_{1}-\lambda_{2}\right) \dot{\varphi} & =\lambda_{2} v_{1}^{\top} F v_{2}+\lambda_{1} v_{1}^{\top} F^{\top} v_{2}+v_{1}^{\top} S v_{2}-\left(\lambda_{1}-\lambda_{2}\right) \dot{u}_{1}^{\top} u_{2} .
\end{aligned}
$$

The matrix $W(t)$ (the required stochastic sensitivity function of the cycle) as a solution of systems (3)-(5) can be obtained via the following limiting procedure.

Theorem 2. Let the T-periodic matrix $W(t)$ be a solution of systems (3)-(5), and let $\lambda_{1}(t), \lambda_{2}(t), \varphi(t)$ be an arbitrary solution of system (8) on the interval $[0,+\infty)$. Define $V(t)=$ $\lambda_{1}(t) \cdot P_{1}(t)+\lambda_{2}(t) \cdot P_{2}(t)$, where $P_{i}(t)=v_{i}(t) v_{i}^{\top}(t)$ with the vector functions $v_{i}(t)$ obtained from $E q$. (7). Then, matrix $V(t)$ tends to matrix $W(t)$ as $t \rightarrow+\infty$,

$$
\lim _{t \rightarrow+\infty}(V(t)-W(t))=0 .
$$

So, the matrix $W(t)$ is determined by the scalar functions $\lambda_{1}(t), \lambda_{2}(t)$ and vectors $v_{1}(t), v_{2}(t)$. In the case of non- degenerate noise, the functions $\lambda_{1}(t), \lambda_{2}(t)$ are strictly positive, and they together determine a dispersion of the random trajectories along the vectors $v_{1}(t), v_{2}(t)$ at any point $\xi(t)$ of the deterministic cycle.

\section{B. Confidence ellipses}

The values of $\lambda_{1}(t), \lambda_{2}(t)$ determine the size, and that of $v_{1}(t), v_{2}(t)$ determine the directions of a dispersion ellipse axis. The equation of this ellipse in a plane $\Pi_{t}$ is as follows:

$$
(x-\xi(t))^{\top} W^{+}(t)(x-\xi(t))=2 k^{2} \varepsilon^{2},
$$

where the parameter $k$ determines a fiducial probability $P=$ $1-e^{-k^{2}}$. It means that random states distributed in the Poincare section $\Pi_{t}$ belong to the interior of this ellipse with the probability $P$. For scalar coordinates $\mu_{i}=\left(x-\xi(t), v_{i}(t)\right)$, the equation of this ellipse can be written in a standard form

$$
\frac{\mu_{1}^{2}}{\lambda_{1}}+\frac{\mu_{2}^{2}}{\lambda_{2}}=2 k^{2} \varepsilon^{2}
$$

It is noted that the method of confidence ellipses has been used for analyzing the excitability of a neuron ${ }^{16}$ and for spatial analysis of 3D-stochastic cycles. ${ }^{37}$

\section{ANALYSIS OF NOISE-INDUCED CHAOS IN THE CHEN SYSTEM}

Consider the stochastically perturbed Chen systems

$$
\begin{aligned}
& \dot{x}=a(-x+y)+\varepsilon \dot{w}_{1}, \\
& \dot{y}=(c-a) x+c y-x z+\varepsilon \dot{w}_{2}, \\
& \dot{z}=-b z+x y+\varepsilon \dot{w}_{3} .
\end{aligned}
$$

Here, $w_{i}(t)$ are independent standard Wiener processes with Gaussian increments, $E\left(w_{i}(t)-w_{i}(s)\right)=0, E\left(w_{i}(t)-w_{i}(s)\right)^{2}$ $=|t-s|$, and parameter $\varepsilon$ is the noise intensity, $i=1,2,3$.

Fix the parameters $a=45$ and $c=28$ within the chaotic region. For $\varepsilon=0$, on the interval $1.5 \leq b \leq 2.9$, the deterministic system (9) demonstrates a well-known infinite chain of period-doubling bifurcations with a transition from order (see Fig. 1(a) for $b=1.5$ ) to chaos (see Fig. 1(d) for $b=2.9)^{38}$

First, we study the system (9) with a fixed $b=1.5$ and a varying noise intensity $\varepsilon$. Recall that an analysis of the stochastic cycles of the Chen system was already reported earlier in Ref. 39.

In Figs. 1(a)-1(c), the $Y O Z$ and $X O Y$ projections of the attractor of the Chen system and time series are plotted for fixed parameters $a, b, c$ and noise intensity values $\varepsilon=0,0.1,0.2$.

The unperturbed Chen system (with $\varepsilon=0$ ) exhibits a deterministic limit cycle corresponding to the periodic solution with a fixed amplitude (see Fig. 1(a)). Here, the $x(t)$ coordinate monotonically oscillates between the amplitude values of \pm 8.7 .

For small noise, random states of stochastic cycle are concentrated in a small vicinity of the deterministic cycle. As the noise intensity increases (see Fig. 1(b) for $\varepsilon=0.1$ ), the dispersion of the random trajectories grows. The 
a)
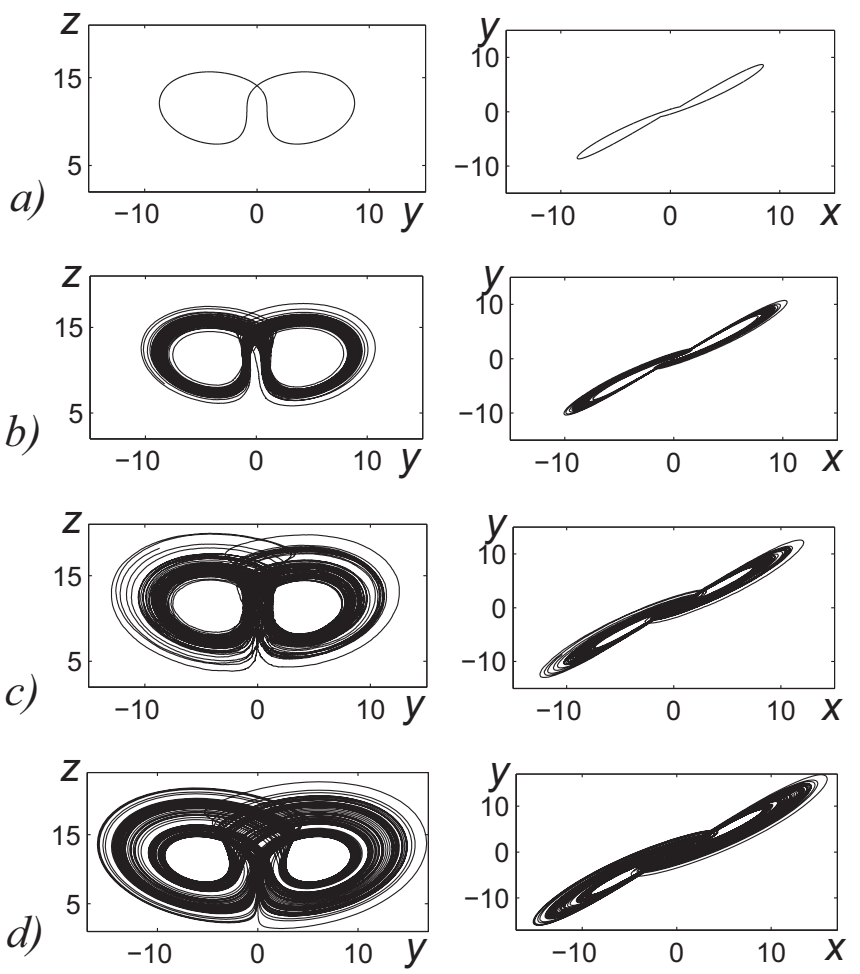
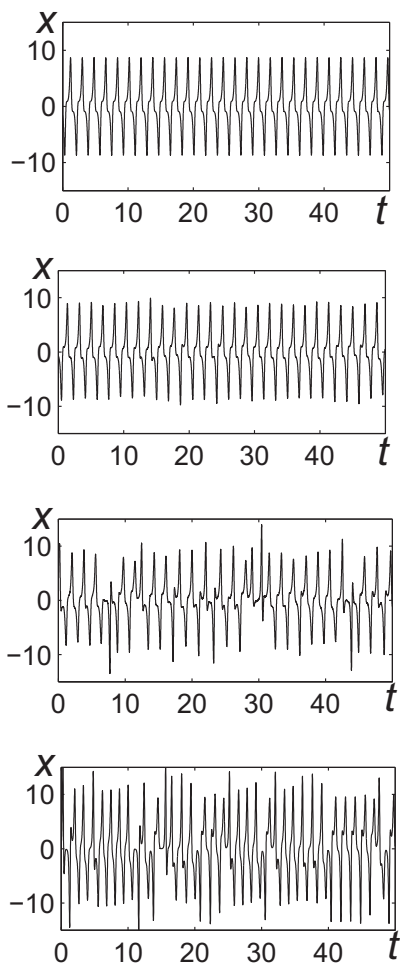

FIG. 1. Attractors and time series of the Chen system with $a=45, c=28$ for $b=1.5$ with noise intensity: (a) $\varepsilon=0$, (b) $\varepsilon=0.1$, (c) $\varepsilon=0.2$, and chaos for (d) $b=2.9, \varepsilon=0$. amplitude values of $x(t)$ show an appreciable random variation, and the small stochastic bursts are observed on the time-intervals between consecutive amplitude values.

With further increase of the noise intensity (see Fig. 1(c) for $\varepsilon=0.2$ ), the random transitions between different portions of the cycle are observed. These transitions are localized in the central part of the attractor, near the point $(0,0,13)$. Here, the $x(t)$-coordinate in a vicinity of the value $x=0$ sharply interrupts the deterministic monotonicity and returns back to the just abandoned amplitude value. This effect intensifies as the noise intensity increases.

Due to the above transitions, the form of the stochastic attractor essentially changes. From a general geometric point of view, these stochastic attractors (see Fig. 1(c)) are similar to the chaotic attractor of the unperturbed Chen system (1) (see Fig. 1(d)). This qualitative change of the form of the stochastic attractor can be interpreted as a noise-induced transition from order to chaos.

Next, we examine whether the chaos-like stochastic attractors (see Fig. 1(c)) can be characterized as true chaotic attractors.

A convenient measure of chaos is the Lyapunov exponent. In Fig. 2, a plot of the largest Lyapunov exponent $\Lambda(\varepsilon)$ is presented. Here, $\Lambda(0)=0$, which is theoretically clear. For small $\varepsilon>0$, the function $\Lambda$ has values close to zero. Beyond some threshold $\varepsilon^{*}$, the values of $\Lambda(\varepsilon)$ are definitely positive. Moreover, the function $\Lambda(\varepsilon)$ monotonically grows up to $\Lambda \approx 0.6$. This value is close to the largest Lyapunov exponent of the chaotic attractor of the deterministic Chen system with $b=2.9$. Thus, the stochastic attractor (see Fig. 1(c)) and the chaotic deterministic attractor (see Fig. 1(d)) are similar, not only from the geometrical point of view but also from their close dynamical characteristics.
We next consider the probabilistic mechanism of this noise-induced phenomenon and analyze it with the help of the stochastic sensitivity functions technique and the confidence ellipses.

Specifically, our attention is focused on the dispersion of the random trajectories around the deterministic cycle. Indeed, the growth of this dispersion is a fundamental mechanism of the noise-induced phenomenon studied here.

The spatial arrangement of the random trajectories can be described by the stochastic sensitivity matrix $W(t)$. Its eigenvalues $\lambda_{1}(t)>\lambda_{2}(t)$ can be used as basic scalar characteristics of the cycle sensitivity. For the stochastic cycle of the Chen system with $b=1.5$, plots of the functions $\lambda_{1}(t), \lambda_{2}(t)$ are shown in Figs. 3(a) and 3(b). One can notice an essential

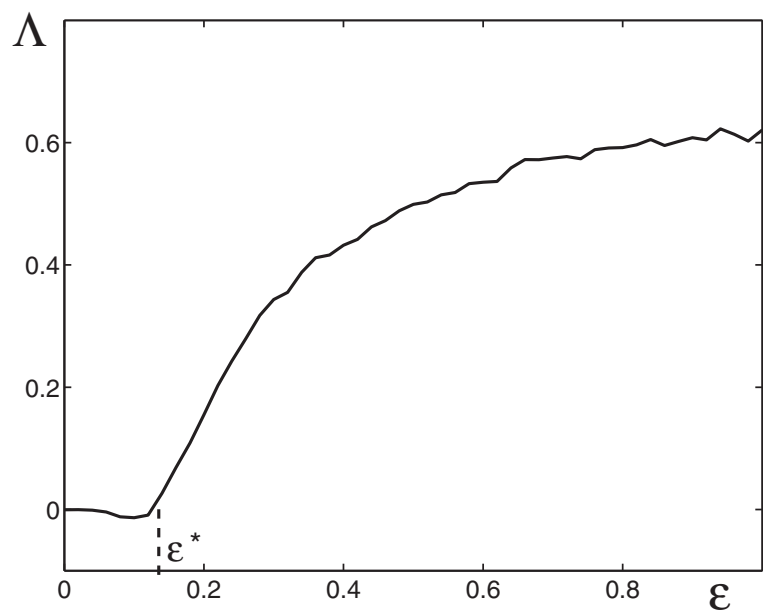

FIG. 2. Largest Lyapunov exponent $\Lambda(\varepsilon)$ of the stochastic Chen system with $b=1.5 ; \varepsilon^{*}$ is the threshold noise intensity. 

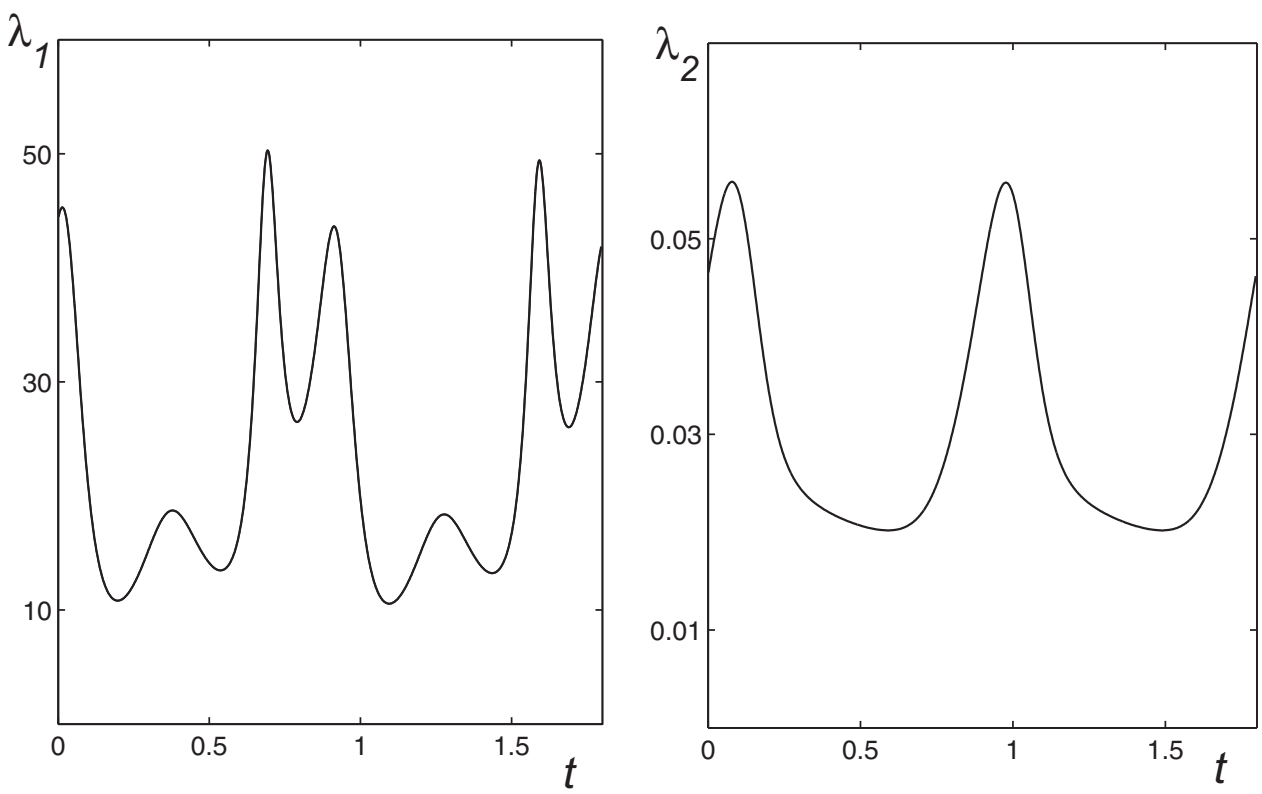

FIG. 3. Stochastic sensitivity functions for the Chen system with $a=45, b=1.5$, and $c=28$. overfall of the values $\lambda_{1}(t), \lambda_{2}(t)$ on the interval $[0, T]$. The values $\lambda_{1}(t)$ are greater than the values of $\lambda_{2}(t)$ by several orders. It confirms a considerable non-uniformity of the random trajectories dispersion.

Confidence ellipses give a visual geometric description of the spatial arrangement of the probabilistic distribution for random states in the Poincaré sections of a stochastic cycle. In Fig. 4, the intersection points of the stochastic trajectories, with a Poincaré plane orthogonal to the deterministic cycle at the point $x=-1.1642, y=-1.2561, z=8.8269$, are plotted by asterisks. Here, $\eta_{1}, \eta_{2}$ are coordinates of the points in the basis $u_{1}, u_{2}$ of this plane. Confidence ellipses for $P=0.8$ (solid line) and $P=0.95$ (dashed line) are also presented. As one can see, these ellipses adequately reflect the main features of the dispersion of the random states.

Now, we use these confidence ellipses to analyze the noise-induced transitions from the regular stochastic cycle to

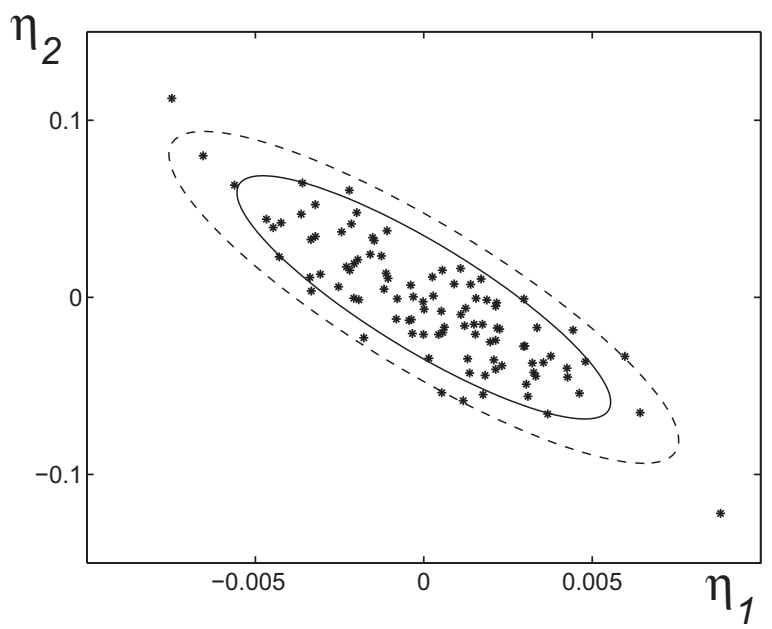

FIG. 4. Confidence ellipses for $b=1.5$, and $P=0.8$ (solid line), $P=0.95$ (dashed line) and intersection points (asterisks) of random trajectories for the orthogonal Poincaré section at the point $x=-1.1642, y=-1.2561$, and $z=8.8269$. the chaos-like stochastic cycle. First, we have to choose a reasonable location of the Poincaré secant plane. We look for a "transition bridge," where random trajectories pass from one part of the cycle to another. As one can see in the above figures, this transition bridge is localized near the point $(0,0,13)$. Therefore, to analyze the noise-induced phenomenon, we use the secant plain $x=0$. Note that the deterministic cycle intersects this plane at two symmetric points, $M_{1,2}(0, \pm 0.4398,13.5349)$. This Poincaré secant plane is localized at the center of the "transition bridge." Here, noiseinduced transitions are observed for smaller noise intensities than for others Poincaré sections.

In Fig. 5, points (asterisks) of the intersection of the random trajectories with the Poincaré plane $x=0$ and confidence ellipses with the fiducial probability $P=0.95$ are plotted for

a)

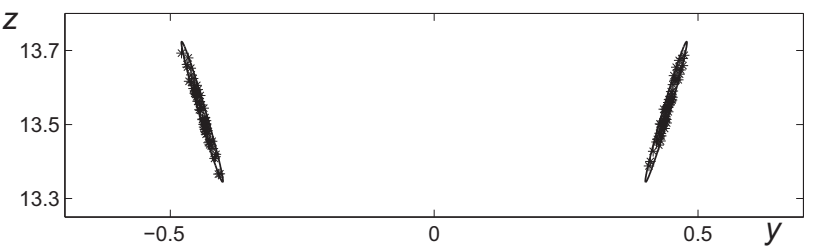

$Z$

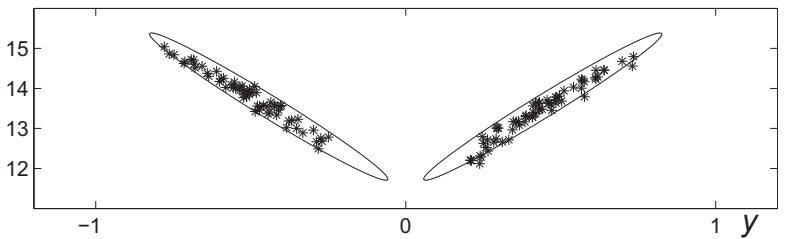

z

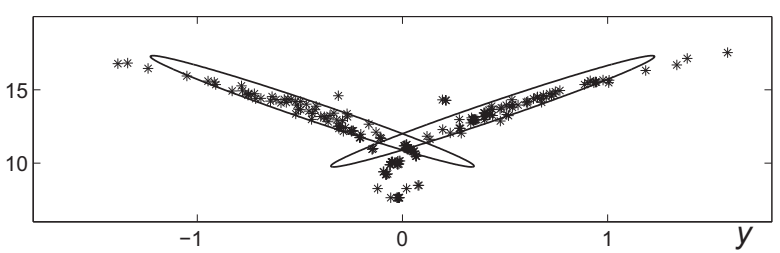

FIG. 5. Dispersion ellipses and intersection points (asterisks) of random trajectories with Poincaré plane $x=0$ for $b=1.5, P=0.95$, and (a) $\varepsilon=0.01$, (b) $\varepsilon=0.1$, (c) $\varepsilon=0.2$. 


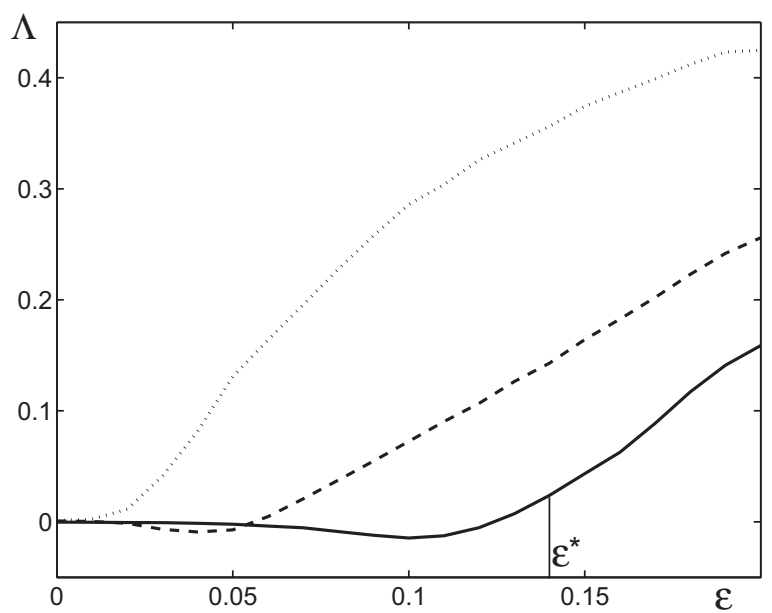

FIG. 6. Largest Lyapunov exponent $\Lambda(\varepsilon)$ for the stochastic Chen system with $b=1.5$ (solid curve), $b=2$ (dashed curve), and $b=2.3$ (dotted curve); $\varepsilon^{*}$ is the threshold noise intensity.

different noise levels. For a sufficiently small noise intensity, the confidence ellipses are localized near the points $M_{1,2}$ of the deterministic cycle. These ellipses are small and definitely separated one from another (see Fig. 5(a), with $\varepsilon=0.01$ ). As the noise intensity increases, the confidence ellipses expand and begin to come close to each other (see Fig. 5(b)). As the parameter $\varepsilon$ further grows, these ellipses approach to each other and begin to intersect (see Fig. 5(c)). This intersection provides an indication for the beginning of the noise-induced transition. Noise intensity that corresponds to the onset of this intersection can be used as an estimation of the threshold value $\varepsilon^{*}$. For the considered parameter set, we have $\varepsilon^{*}=0.14$. Note that the results of the direct numerical simulations (asterisks) agree with our theoretical prediction based on stochastic sensitivity functions method and ellipses technique.
The intersection of ellipses for $\varepsilon>\varepsilon^{*}$ means that the random trajectories pass from one portion of the cycle to another with a high probability. These transitions essentially change the dynamics of the Chen system from regular to chaotic. Indeed, near the value $\varepsilon^{*}=0.14$, the largest Lyapunov exponent $\Lambda(\varepsilon)$ becomes positive (see Figs. 2 and 6) and marks the onset of chaos. As noise intensity increases, $\Lambda(\varepsilon)$ increases too. For $\varepsilon=0.2$, the largest Lyapunov exponent is $\Lambda=0.17$.

Now, we consider how the stochastic sensitivity functions technique and method of dispersion ellipses can be applied to noise-induced chaotization, for the case of parameter $b>1.5$ within the period-doubling bifurcation zone.

In Fig. 6, the function $\Lambda(\varepsilon)$ for three different values of $b$ is plotted. As can be seen, for $b=2$ (dashed line) and $b=2.3$ (dotted line), the largest Lyapunov exponent becomes positive for much smaller values of $\varepsilon$ than in the case of $b=1.5$ (solid line). The value of $\Lambda>0$ is a measure of chaos for the considered attractors.

Let us fix the chaos threshold value $\bar{\Lambda}=0.01$ (weak chaos). A condition of $\Lambda(\varepsilon)>\bar{\Lambda}$ is satisfied if $\varepsilon>0.13$ for $b=1.5, \varepsilon>0.07$ for $b=2$, and $\varepsilon>0.02$ for $b=2.3$. So the noise intensity $\varepsilon$ that generates "weak chaos" depends on the value of the parameter $b$ substantially. The underlying mechanism of this dependence is due to the variation of $b$ which implies changes in both the geometry of the initial deterministic attractors and its stochastic sensitivity.

Next, consider the most essential issues in analyzing the noise-induced chaotization for more complicated deterministic attractor with $b=2.3$. With $b=2.3$, the deterministic Chen system $(\varepsilon=0)$ has two coexisting non-symmetric 2-cycles (see Fig. 7(a)). The corresponding stochastic attractors for $\varepsilon=0.005, \varepsilon=0.04, \varepsilon=0.08$ are plotted in Figs. 7(b)-7(d), respectively. In Figs. 8(a)-8(c), along with dispersion ellipses, intersections of these stochastic attractors with the Poincaré plane $x=0$ are shown. These ellipses were
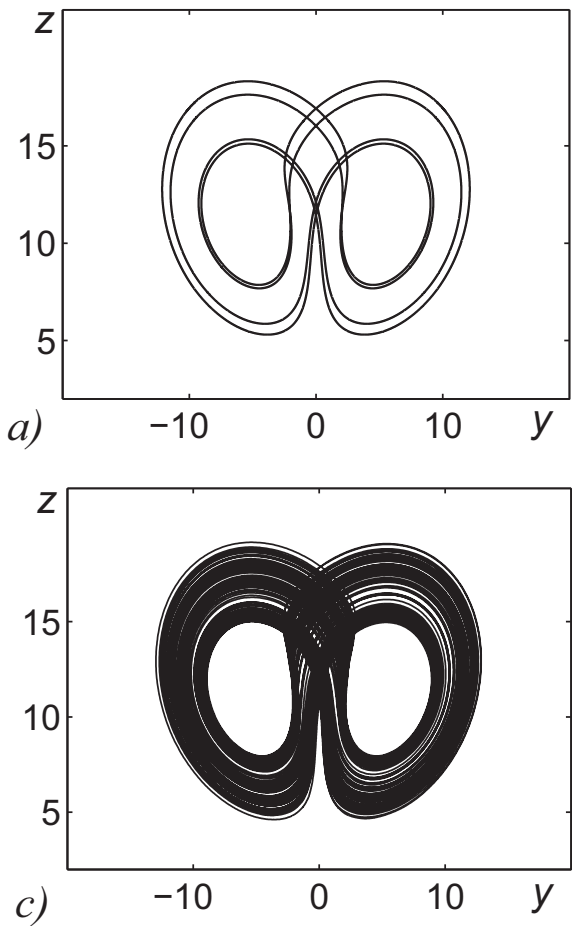
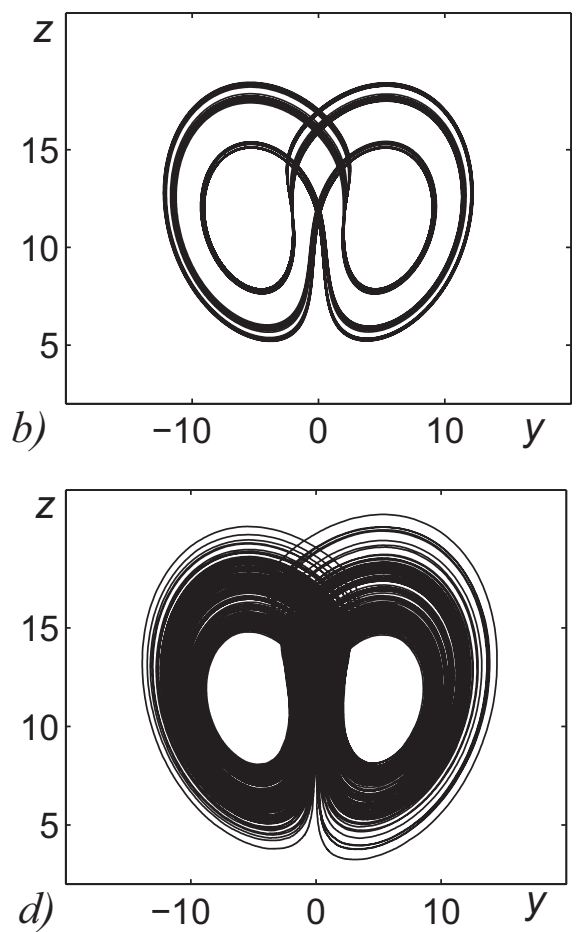

FIG. 7. Attractors of the Chen system with $a=45, b=2.3$, and $c=28$ for noise intensity: (a) $\varepsilon=0$, (b) $\varepsilon=0.005$, (c) $\varepsilon=0.04$, and $(\mathrm{d}) \varepsilon=0.08$. 

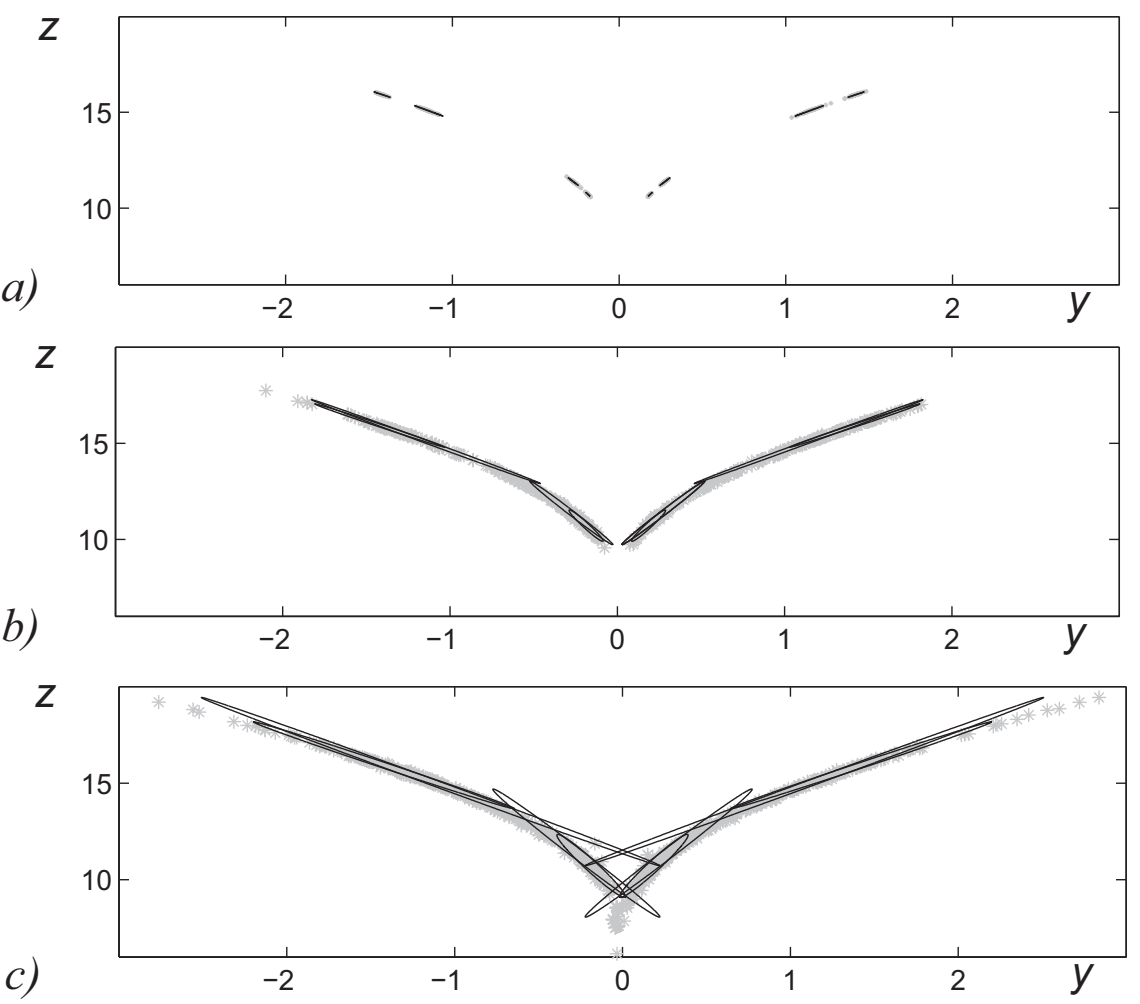

FIG. 8. Dispersion ellipses and intersection points (grey asterisks) of random trajectories with Poincaré plane $x=0$ for $b=2.3, P=0.95$, and (a) $\varepsilon=0.005$, (b) $\varepsilon=0.04$, (c) $\varepsilon=0.08$. calculated with the help of the stochastic sensitivity function technique for the fiducial probability $P=0.95$.

For small noise with $\varepsilon=0.005$, stochastically perturbed trajectories are localized near the deterministic attractor. Sets of random states in the Poincaré section $x=0$ are well separated. Dispersion ellipses do not intersect each other. Here, we observe two stochastic 2-cycles (see Fig. 8(a)). As the noise intensity increases (to $\varepsilon=0.04$ ), the noise-induced transitions between close portions of the 2-cycle emerge and sets of random states coalesce to a single set in pairs. Dispersion ellipses intersect each other (see Fig. 8(b)). As a result, each of the stochastic 2-cycles transforms into a stochastic 1-cycle. This phenomenon, the so-called backward stochastic bifurcation, has been studied earlier in Ref. 31 for the stochastic Roessler model. These backward stochastic bifurcations are associated with changes of the dynamic properties of the stochastic attractor. For the considered noise values, we have $\Lambda(0.005)<\bar{\Lambda}<\Lambda(0.04)=0.08$. It means that the noise intensity $\varepsilon=0.04$ transforms the regular attractor to a chaotic one.

As the noise intensity increases further, the dispersion ellipses continue to expand. These ellipses cross the separation line $y=0$ and, for $\varepsilon=0.08$, begin to intersect with each other (see Fig. 8(c)). This type of intersections is similar to the case for $b=1.5$ considered above (see Fig. 5(b)). Here, for $b=2.3$, the largest Lyapunov exponent equals $\Lambda=0.23$. So, for $b=2.3$ chaos occurs for considerably smaller noise intensity values as compared to the case of for $b=1.5$.

\section{CONCLUSION}

Under random disturbances, a phase trajectory can demonstrate a noise-induced hopping between different but close portions of the stochastic cycle. During this hopping, the ran- dom trajectories of Chen system with a high probability fall within zones of divergency (local instability) and the dynamics of the perturbed system as a whole becomes chaotic. In this paper, our technique of confidence ellipses is used for the constructive analysis of the noise-induced hopping through zones of local instability. In fact, both the size and the spatial arrangement of dispersion ellipses are defined by the stochastic sensitivity of the cycles and noise intensity. As noise intensity increases, these ellipses are enlarged and begin to occupy some zones where trajectories diverge and the chaotization starts. So, our technique allows us to effectively estimate the threshold noise intensity corresponding the main features of the noise-induced transitions from regularity to chaos, without using costly direct numerical simulations on the random trajectories, which is inevitable when applying the method of Lyapunov exponents. Note that the method of Lyapunov exponents only identifies the existence of chaos a posteriori, after extensive numerical simulations on the random trajectories, but our method of dispersion ellipses allows one to predict the transition to chaos a priori without performing such extensive simulations.

\section{ACKNOWLEDGMENTS}

G.C. acknowledges the Hong Kong Research Grants Council for the GRF Grant CityU 1117/10E. I.B. and L.R. were partially supported by the RFBR Grant 10-0196022ural and Federal Target Program 1.1099.2011.

\section{APPENDIX: NUMERICAL PROCEDURES AND ALGORITHMS}

Rewrite an initial unforced deterministic system $\dot{x}=$ $f(x)$ and its $T$-periodic solution $x=\xi(t)$ with exponentially stable phase curve $\gamma$ (limit cycle) for $n=3$ by coordinates 


$$
\begin{aligned}
& \dot{x}_{1}=f_{1}\left(x_{1}, x_{2}, x_{3}\right), \quad \dot{x}_{2}=f_{2}\left(x_{1}, x_{2}, x_{3}\right), \\
& \dot{x}_{3}=f_{3}\left(x_{1}, x_{2}, x_{3}\right), \quad \xi(t)=\left(\xi_{1}(t), \xi_{2}(t), \xi_{3}(t)\right)^{\top} .
\end{aligned}
$$

To calculate SSF, we use the following algorithm:

(1) Find the limit cycle $\gamma$ with required accuracy. For the coordinates $\xi_{j}(t)(j=1,2,3)$ of the $T$-periodic solution, the discretization is as follows:

$$
\xi_{j, i} \approx \xi_{j}\left(t_{i}\right), \quad t_{i}=i h, \quad i=0,1, \ldots, N, \quad h=\frac{T}{N} .
$$

We used a numerical Runge-Kutta 4th order scheme with time step $h \sim 0.0001$.

(2) For computation the decompositions (6) and (7) through the system (8), it is necessary to calculate parameters $F\left(t_{i}\right), S\left(t_{i}\right), u_{1}\left(t_{i}\right), u_{2}\left(t_{i}\right), \dot{u}_{1}\left(t_{i}\right)$. Matrices $F, S$ are determined by the formula

$$
F\left(t_{i}\right)=\frac{\partial f}{\partial x}\left(\xi\left(t_{i}\right)\right), \quad S\left(t_{i}\right)=\sigma\left(\xi\left(t_{i}\right)\right) \sigma^{\top}\left(\xi\left(t_{i}\right)\right) .
$$

To calculate vector functions $u_{1}(t), u_{2}(t), \dot{u}_{1}(t)$, the following method is used. A tangent vector $r(t)=\left(f_{1}(t)\right.$, $\left.f_{2}(t), f_{3}(t)\right)^{\top}$ has coordinates $f_{j}(t)=f_{j}(\xi(t)), j=1,2,3$. The vectors $u_{1}(t), u_{2}(t)$ of orthonormal basis of a plane $\Pi_{t}$ can be found in the following form (variable $t$ is omitted)

$$
u_{1}=g_{1} \cdot\left(\begin{array}{c}
-f_{2} \\
f_{1} \\
0
\end{array}\right), u_{2}=g_{2} \cdot\left(\begin{array}{c}
-f_{1} f_{3} \\
-f_{2} f_{3} \\
f_{1}^{2}+f_{2}^{2}
\end{array}\right),
$$

where

$g_{1}=\left(f_{1}^{2}+f_{2}^{2}\right)^{-\frac{1}{2}}, \quad g_{2}=\left(f_{3}^{2}\left(f_{1}^{2}+f_{2}^{2}\right)+\left(f_{1}^{2}+f_{2}^{2}\right)^{2}\right)^{-\frac{1}{2}}$.

As a result, the following formula holds

$$
\begin{aligned}
\dot{u}_{1}=\dot{g}_{1} \cdot\left(\begin{array}{c}
-f_{2} \\
f_{1} \\
0
\end{array}\right)+g_{1} \cdot\left(\begin{array}{ccc}
-\frac{\partial f_{2}}{\partial x_{1}} & -\frac{\partial f_{2}}{\partial x_{2}} & -\frac{\partial f_{2}}{\partial x_{3}} \\
\frac{\partial f_{1}}{\partial x_{1}} & \frac{\partial f_{1}}{\partial x_{2}} & \frac{\partial f_{1}}{\partial x_{3}} \\
0 & 0 & 0
\end{array}\right) \\
\cdot\left(\begin{array}{c}
f_{1} \\
f_{2} \\
f_{3}
\end{array}\right),
\end{aligned}
$$

where

$$
\begin{aligned}
\dot{g}_{1}= & -\left(f_{1}^{2}+f_{2}^{2}\right)^{-\frac{3}{2}} \cdot\left(f_{1} \cdot\left(\frac{\partial f_{1}}{\partial x_{1}} f_{1}+\frac{\partial f_{1}}{\partial x_{2}} f_{2}+\frac{\partial f_{1}}{\partial x_{3}} f_{3}\right)\right. \\
& \left.+f_{2} \cdot\left(\frac{\partial f_{2}}{\partial x_{1}} f_{1}+\frac{\partial f_{2}}{\partial x_{2}} f_{2}+\frac{\partial f_{2}}{\partial x_{3}} f_{3}\right)\right) .
\end{aligned}
$$

(3) For the computation of $T$-periodic solution $\left(\lambda_{1}(t), \lambda_{2}(t)\right.$, $\left.\varphi(t), v_{1}(t), v_{2}(t)\right)$ of systems (7) and (8), we use a stabilization method (see Theorem 2) and Runge-Kutta 4 th order scheme. The rate of convergency of stabilization method is determined by the degree of stability of the limit cycle.

(4) Stochastic sensitivity matrix $W(t)$ can be found from Eq. (6) through $\lambda_{1}(t), \lambda_{2}(t), v_{1}(t), v_{2}(t)$ calculated above.

(5) To calculate a dispersion ellipse, we use the explicit parametrical formulas:

$\eta_{1}=\varepsilon \sqrt{-2 \ln (1-P)}\left(\sqrt{\lambda_{1}} \cos \theta \cos \varphi-\sqrt{\lambda_{2}} \sin \theta \sin \varphi\right)$

$\eta_{2}=\varepsilon \sqrt{-2 \ln (1-P)}\left(\sqrt{\lambda_{1}} \cos \theta \sin \varphi+\sqrt{\lambda_{2}} \sin \theta \cos \varphi\right)$.

Here, $\eta_{1}, \eta_{2}$ are the coordinates of the ellipse in terms of the basis $u_{1}, u_{2}$, and $\theta$ is a parameter of this ellipse, satisfying $0 \leq \theta \leq 2 \pi$.

For the calculation of the largest Lyapunov exponent, a standard Benettin method ${ }^{40,41}$ utilizing the variation equations was used. Runge-Kutta 4th order scheme with step 0.001 and averaging time $10^{5}$ provides two true significant digits.

${ }^{1}$ F. Moss and P. V. E. McClintock, Noise in Nonlinear Dynamical Systems (Cambridge University Press, Cambridge, 2007).

${ }^{2}$ V. S. Anishchenko, V. V. Astakhov, A. B. Neiman, T. E. Vadivasova, and L. Schimansky-Geier, Nonlinear Dynamics of Chaotic and Stochastic Systems. Tutorial and Modern Development (Springer-Verlag, Berlin, 2007).

${ }^{3} \mathrm{~W}$. Horsthemke and R. Lefever, Noise-Induced Transitions (Springer, Berlin, 1984).

${ }^{4}$ P. S. Landa and P. V. E. McClintock, Phys. Rep. 323, 1 (2000).

${ }^{5}$ L. Arnold, Random Dynamical Systems (Springer-Verlag, Berlin, 1998).

${ }^{6} \mathrm{R}$. Lefever and J. Turner, Phys. Rev. Lett. 56, 1631 (1986).

${ }^{7}$ K. Malick and P. Marcq, Eur. Phys. J. 36, 119 (2003).

${ }^{8}$ L. Gammaitoni, P. Hanggi, P. Jung, and F. Marchesoni, Rev. Mod. Phys. 70, 223 (1998).

${ }^{9}$ M. D. McDonnell, N. G. Stocks, C. E. M. Pearce, and D. Abbott, Stochastic Resonance: From Suprathreshold Stochastic Resonance to Stochastic Signal Quantization (Cambridge University Press, Cambridge, 2008).

${ }^{10} \mathrm{~K}$. Matsumoto and I. Tsuda, J. Stat. Phys. 31, 87 (1983).

${ }^{11}$ F. Gassmann, Phys. Rev. E 55, 2215 (1997).

${ }^{12}$ J. B. Gao, S. K. Hwang, and J. M. Liu, Phys. Rev. Lett. 82, 1132 (1999).

${ }^{13}$ S. Ellner and P. Turchin, Oikos 111, 620 (2005).

${ }^{14}$ Y.-C. Lai and T. Tel, Transient Chaos. Complex Dynamics on Finite Time Scales, Applied Mathematical Sciences Vol. 173 (Springer, 2011).

${ }^{15}$ B. Lindner, J. Garcia-Ojalvo, A. Neiman, and L. Schimansky-Geier, Phys. Rep. 392, 321 (2004).

${ }^{16}$ L. Ryashko and I. Bashkirtseva, Phys. Rev. E 83, 061109 (2011).

${ }^{17}$ R. J. Reategui and A. N. Pisarchik, Phys. Rev. E 69, 067203 (2004).

${ }^{18}$ S. Aumaitre, K. Mallick, and F. Pétrélis, J. Stat. Mech.: Theory Exp. 2007, P07016 (2007).

${ }^{19}$ M. Pradas, D. Tseluiko, S. Kalliadasis, D. T. Papageorgiou, and G. A. Pavliotis, Phys. Rev. Lett. 106, 060602 (2011).

${ }^{20}$ J. C. Sommerer, E. Ott, and C. Grebogi, Phys. Rev. A 43, 1754 (1991).

${ }^{21}$ V. S. Anishchenko and M. E. Khairulin, Tech. Phys. Lett. 37, 561 (2011).

${ }^{22}$ A. S. Pikovsky and J. Kurths, Phys. Rev. Lett. 78, 775 (1997).

${ }^{23}$ J. B. Gao, W. W. Tung, and N. S. V. Rao, Phys. Rev. Lett. 89, 254101 (2002).

${ }^{24}$ C. B. Muratov and E. Vanden-Eijnden, Chaos 18, 015111 (2008).

${ }^{25}$ Y.-C. Lai, Z. Liu, L. Billings, and I. B. Schwartz, Phys. Rev. E 67, 026210 (2003).

${ }^{26}$ F. Arecchi, R. Badii, and A. Politi, Phys. Rev. A 32, 402 (1985).

${ }^{27}$ S. Kraut and U. Feudel, Phys. Rev. E 66, 015207 (2002).

${ }^{28}$ W.-W. Tung, J. Hu, J. Gao, and V. Billock, Int. J. Bifurcation Chaos Appl. Sci. Eng. 18, 1749 (2008).

${ }^{29}$ G. Mayer-Kress and H. Haken, J. Stat. Phys. 26, 149 (1981).

${ }^{30}$ J. P. Crutchfeld, J. D. Farmer, and B. A. Huberman, Phys. Rep. 92, 45 (1982). 
${ }^{31}$ I. Bashkirtseva, L. Ryashko, and P. Stikhin, Fluct. Noise Lett. 9, 89 (2010).

${ }^{32}$ T. Tel and J.-C. Lai, Phys. Rev. E 81, 056208 (2010).

${ }^{33}$ I. Bashkirtseva and L. Ryashko, Phys. Rev. E 79, 041106 (2009).

${ }^{34}$ S. Čelikovský and G. Chen, Int. J. Bifurcation Chaos Appl. Sci. Eng. 12, 1789 (2002).

${ }^{35}$ M. I. Freidlin and A. D. Wentzell, Random Perturbations of Dynamical Systems (Springer, Berlin, 1984).

${ }^{36}$ I. A. Bashkirtseva and L. B. Ryashko, Math. Comput. Simul. 66, 55 (2004).
${ }^{37}$ L. Ryashko, I. Bashkirtseva, A. Gubkin, and P. Stikhin, Math. Comput. Simul. 80, 256 (2009).

${ }^{38}$ T. Ueta and G. Chen, Int. J. Bifurcation Chaos Appl. Sci. Eng. 10, 1917 (2000).

${ }^{39}$ I. Bashkirtseva, G. Chen, and L. Ryashko, Int. J. Bifurcation Chaos Appl. Sci. Eng. 20, 1439 (2010).

${ }^{40}$ G. Benettin, L. Galgani, A. Giorgilli, and J.-M. Strelcyn, Meccanica 15, 21 (1980).

${ }^{41}$ C. Skokos, Lect. Notes Phys. 790, 63 (2010). 